ARTICLE

https://doi.org/10.1038/s41467-019-13197-z

\title{
Band splitting with vanishing spin polarizations in noncentrosymmetric crystals
}

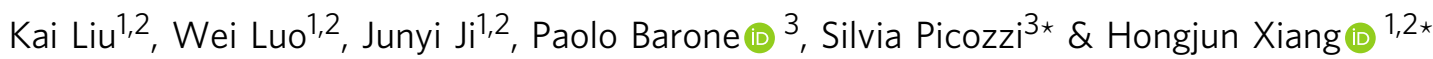

The Dresselhaus and Rashba effects are well-known phenomena in solid-state physics, in which spin-orbit coupling splits spin-up and spin-down energy bands of nonmagnetic noncentrosymmetric crystals. Here, we discuss a phenomenon we dub band splitting with vanishing spin polarizations (BSVSP), in which, as usual, spin-orbit coupling splits the energy bands in nonmagnetic non-centrosymmetric systems. Surprisingly, however, both split bands show no net spin polarization along certain high-symmetry lines in the Brillouin zone. In order to rationalize this phenomenon, we propose a classification of point groups into pseudo-polar and non-pseudo-polar groups. By means of first-principles simulations, we demonstrate that BSVSP can take place in both symmorphic (e.g., bulk GaAs) and non-symmorphic systems (e.g., two dimensional ferroelectric SnTe). Furthermore, we identify a linear magnetoelectric coupling in reciprocal space, which could be employed to tune the spin polarization with an external electric field. The BSVSP effect and its manipulation could therefore form the basis for future spintronic devices.

\footnotetext{
${ }^{1}$ Key Laboratory of Computational Physical Sciences (Ministry of Education), State Key Laboratory of Surface Physics, and Department of Physics, Fudan University, Shanghai 200433, China. ${ }^{2}$ Collaborative Innovation Center of Advanced Microstructures, Nanjing 210093, China. ${ }^{3}$ Consiglio Nazionale delle Ricerche CNR-SPIN Via dei Vestini 31,66100 Chieti, Italy. *email: silvia.picozzi@spin.cnr.it; hxiang@fudan.edu.cn
} 
$\mathrm{T}$ he study of a relativistic interaction, i.e., the spin-orbit coupling (SOC), has been one of the central themes in the context of spintronics, branch of electronics aiming at utilizing the electron's spin degree of freedom for device applications ${ }^{1}$. Many intriguing SOC-related phenomena were observed, such as spin relaxation ${ }^{2,3}$, optical spin orientation ${ }^{4}$, spin Hall effects ${ }^{5-8}$, persistent spin structures ${ }^{9-11}$, hidden spin polarization in centrosymmetric systems $\mathrm{s}^{12}$, and the spin galvanic effects $^{13,14}$. In particular, Dresselhaus ${ }^{15}$ and Rashba ${ }^{16-18}$ demonstrated that SOC splits spin-up and spin-down bands in nonmagnetic systems lacking inversion symmetry, inducing an effective magnetic field that depends on the crystal momentum $\mathbf{k}$. Although the Dresselhaus and Rashba effects lead to different spin polarizations of the energy bands, they result in a similar band dispersion: the original spin degenerate bands split so that spin-up and spin-down bands shift towards opposite directions in $\mathrm{k}$-space, as shown in Fig. 1. Even though the Rashba effect is generally associated to heterostructures and surfaces, as due to $2 \mathrm{D}$ structural inversion asymmetry, a giant Rashba effect has been recently observed in bulk polar/ferroelectric systems ${ }^{19-23}$. Sometimes, Dresselhaus and Rashba terms coexist ${ }^{24}$, giving rise to interesting physics and applications. For example, if the magnitudes of Dresselhaus and Rashba terms are equal, the spin-orbit field is unidirectional, resulting in a momentum-independent spin configuration (i.e., the so-called "persistent spin texture"), which could support an extraordinarily long spin lifetime of carriers9. To further develop the field of spin-orbitronics (a branch of spintronics) ${ }^{25}$, it is of great interest to explore SOCrelated phenomena beyond the well-known Dresselhaus-Rashba effects. Indeed, a first contribution in this direction has been recently provided by a careful analysis of bulk and site symmetries of crystalline materials, that allowed for a unified description of spin-splitting effects and, more importantly, to unveil hidden spin-polarization phenomena in centrosymmetric systems ${ }^{12}$, recently observed in several layered compounds ${ }^{26-32}$.

In this work, we show that band splitting with vanishing spin polarizations (BSVSP) in acentric nonmagnetic materials, as shown in Fig. 1. The group theory analysis indicates that the band splitting arises from the breaking of inversion symmetry and the vanishing spin polarization (i.e., the expectation value of spin evaluated over each Bloch wavefunction) is due to the presence of additional symmetries. We demonstrate in details why BSVSP is possible and how to achieve it in two prototypical examples, namely bulk GaAs and two-dimensional ferroelectric SnTe, complementing the symmetry analysis with first-principles calculations.

\section{Results}

Group theory analysis on BSVSP. To facilitate our discussion, we first classify the 32 crystallographic point groups into pseudopolar and non-pseudo-polar point groups (Fig. 2), where a pseudo-vector (such as spin, magnetization, angular moment, magnetic field) is allowed or forbidden, respectively. This classification is in the same spirit as the classification of the point groups into polar and non-polar point groups. As we will show below, it will be very useful for checking the existence of bulk and local spin polarizations. We note that spin vectors $S_{x}, S_{y}$, and $S_{z}$

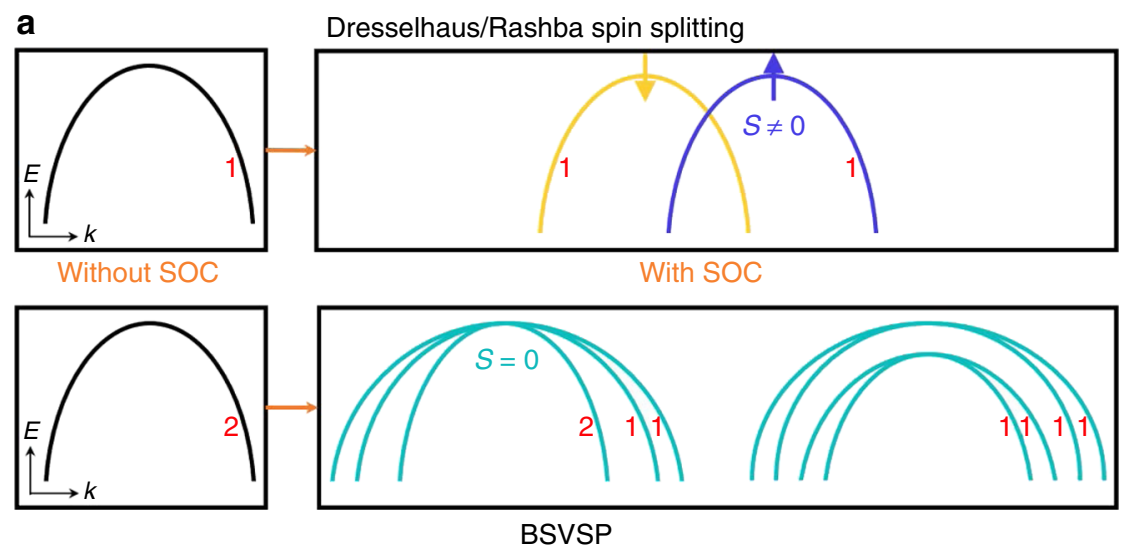

b

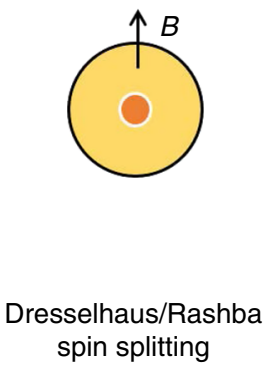

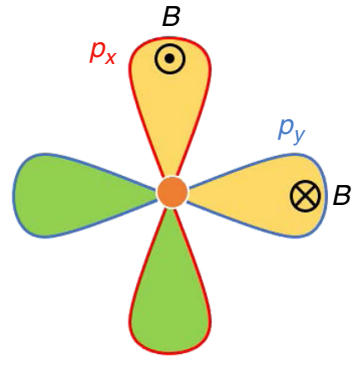

BSVSP - I

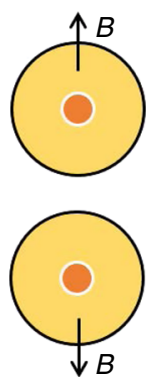

BSVSP - II

Fig. 1 The phenomena and mechanisms of Dresselhaus/Rashba spin splitting and BSVSP. a Schematic depiction of Dresselhaus/Rashba spin splitting and BSVSP. "1" and "2" means single and double degenerate, respectively. In the Dresselhaus/Rashba spin splitting case, a non-degenerate band (without considering spin) splits into two bands with opposite nonzero spin polarizations. In the case of BSVSP, a double degenerate band (without considering spin) splits, resulting in some non-degenerate bands without net spin polarizations (see Supplementary Note 1 for more discussions). b Schematic illustration of the difference between Dresselhaus/Rashba spin splitting and BSVSP. In the case of Dresselhaus/Rashba spin splitting, a k-dependent magnetic field induces the band splitting. In BSVSP-I (II), the magnetic fields acting on two different $p$ orbitals of the same atom (acting on different atoms of the same kind) are equal in strength but opposite, resulting in a band splitting with vanishing spin polarization 


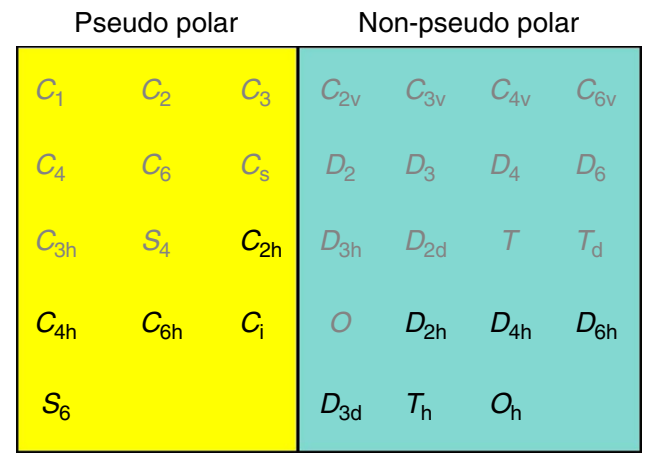

Fig. 2 Classification of 32 crystal point groups into pseudo-polar and nonpseudo-polar point groups. The point group symbols labeled by gray (black) color are non-centrosymmetric (centrosymmetric)

Table 1 Symmorphic space groups which might host BSVSP

\begin{tabular}{lll}
$\begin{array}{l}\text { Crystal } \\
\text { point groups }\end{array}$ & $\begin{array}{l}\text { Symmorphic } \\
\text { space groups }\end{array}$ & High symmetry lines \\
\hline $3 m\left(C_{3 \mathrm{v}}\right)$ & $P 3 m 1, P 31 m, R 3 m$ & $(0,0, u)$ \\
$\overline{6} m 2\left(D_{3 \mathrm{~h}}\right)$ & $P 6 m m$ & $(1 / 3,1 / 3, u)$ \\
$6 m m\left(C_{6 \mathrm{v}}\right)$ & $P \overline{6} m 2, P \overline{6} 2 m$ & $(0,0, u),(1 / 3,1 / 3, u)$ \\
$\overline{4} 3 m\left(T_{d}\right)$ & $P \overline{4} 3 m, F \overline{4} 3 m, \overline{4} 3 m$ & $(u, u, u)$
\end{tabular}

The corresponding crystal point groups and high symmetry lines at which BSVSP occurs are also listed. Here, "u" characterizes the position of the $k$-point in the high symmetry line

will not belong to the identical representation for a non-pseudopolar point group.

The essence of BSVSP manifests in a non-degenerate band at a given crystal momentum $\mathbf{k}$ lacking net spin polarizations in an inversion-asymmetry crystal, as shown in Fig. 1. As such, the BSVSP effect cannot be realized in nonmagnetic centrosymmetric crystals, where Kramers theorem implies that all bands are at least two-fold degenerate (spin degeneracy). Note that BSVSP does not mean the Bloch states are spinless since the expectation value of $S^{2}$ is not zero. In order to realize BSVSP at a given momentum $\mathbf{k}$, two conditions should be simultaneously satisfied: (1) The eigenstate-including SOC-should be non-degenerate, suggesting that the little space group associated with $\mathbf{k}$ should possess at least one 1D double-valued IR; (2) The little point group associated with $\mathbf{k}$ should be a non-pseudo-polar point group. Detailed analysis based on group theory (see Supplementary Note 2) suggests that BSVSP can not only occur in systems with symmorphic space groups, but also in systems with nonsymmorphic space groups. In Table 1, we list all the possible 4 symmorphic space groups which might display BSVSP. The corresponding high-symmetry lines where BSVSP takes place are also given. For a given non-symmorphic space group, one first finds out the possible k-points on the Brillouin zone boundary where the corresponding little point group has at least one 1D double-valued IRs. Then, among these k-points, one finds out the subset of the k-points whose corresponding little point group is non-pseudo-polar. To detect BSVSP in experiment, one can use, for example, spin-polarized angle-resolved photoelectron spectroscopy ${ }^{26}$ to measure the spin polarizations of the bands along a special high symmetry line.

Although the net spin polarizations are zero for both split bands in the BSVSP effect, a local spin polarization might exist, in loose analogy with the hidden spin-polarization effect. By projecting the spin polarization on atomic sites in real space, it is possible to evaluate the contribution of magnetization of each atom to a chosen band. The magnetization of each atom must
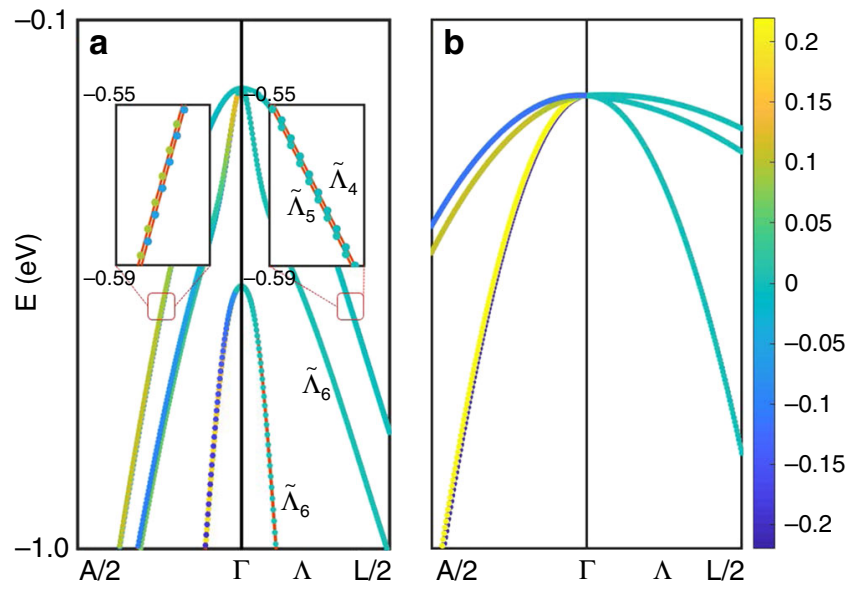

Fig. 3 BSVSP in bulk GaAs. a Band structure and the $x$ component of spin polarizations of bulk GaAs calculated by DFT. The $y$ and $z$ components have the same behavior. The coordinates of symmetry points $A$ and $L$ are $(0.5$, $0.25,0)$ and $(0.5,0.5,0.5)$, respectively. The irreducible representation $\tilde{\Lambda}_{4}$ and $\tilde{\Lambda}_{5}$ are one dimensional. The irreducible representation $\tilde{\Lambda}_{6}$ is two dimensional. $\mathbf{b}$ Band structure and the $x$ component of spin polarizations of bulk GaAs calculated by the $k \cdot p$ Hamiltonian

satisfy the symmetry of the intersection of the site symmetry group and little point group of the considered momentum $\mathbf{k}$. If the resulting symmetry group is non-pseudo-polar, the local spin polarizations also vanish on the chosen atom. Otherwise, local spin polarizations could survive. We find that both situations can occur [GaAs (see below) and $\mathrm{SnI}_{4}$ (see Supplementary Note 4) are representative of the two cases, respectively].

To prove the existence of BSVSP, we calculated from first principles the band structures and related spin polarizations of bulk GaAs and 2D SnTe, representative of symmorphic and nonsymmorphic case, respectively.

BSVSP in zinc-blend GaAs. Bulk GaAs shows a zinc-blende structure with $\bar{F} 43 m$ space group whose corresponding point group is $T_{\mathrm{d}}$. There is no inversion symmetry in GaAs, but since the crystallographic point group is non-polar, it may exhibit Dresselhaus effect (D-1, according to the classification given in ref. ${ }^{12}$ ). In Fig. 3a, we show the calculated SOC band structures and the $x$ components of spin polarizations dependence on $k$ in the vicinity of the $\Gamma$ point for GaAs. The $y$ and $z$ components of spin polarizations have the same behavior. It is well-known that the top valence bands are four-fold degenerate at $\Gamma$. Along symmetry line $\Lambda[\Gamma \rightarrow \mathrm{L}(0.5,0.5,0.5)]$, the top valence bands split into two non-degenerate bands (labeled by $\tilde{\Lambda}_{4}$ and $\tilde{\Lambda}_{5}$, respectively) and a two-fold degenerate band (labeled by $\tilde{\Lambda}_{6}$ ). Note that the two-fold degenerate bands are composed by spin-up and spindown components, resulting in a zero net spin polarization. Interestingly and surprisingly, the top two non-degenerate bands also have vanishing spin polarization, i.e., displaying the BSVSP behavior. Note that all bands are at least two-fold degenerate for isostructural bulk $\mathrm{Si}$ with the inversion symmetry. This means that inversion asymmetry leads to the splitting of the top valence band in GaAs. It should be noted that BSVSP takes place only along special high-symmetry lines. In fact, conventional Dresselhaus splitting with spin polarization takes place for other symmetry lines [e.g., symmetry line $\Gamma \rightarrow \mathrm{A}(0.5,0.25,0)$, as shown in Fig. 3a].

The vanishing spin polarizations can be rationalized by considering the band symmetry properties. Along the $\Lambda$ symmetry line, the little point group is $C_{3 \mathrm{v}}$. According to our previous analysis, $C_{3 \mathrm{v}}$ is classified as non-pseudo-polar. 
Therefore, the $x, y$, and $z$ components of spin polarizations must vanish. Here, we illustrate why the spin polarization along the symmetry line $\Lambda$ should vanish in another clear way. Adopting a $x y z$-coordinate system with the local $\mathrm{z}$-axis along the [111] direction of the cubic cell of bulk GaAs, the point group $C_{3 \mathrm{v}}$ has six symmetry operations, including a mirror symmetry $M_{1}$ which consists of reflection about the $y=0$ plane

$$
M_{1}:\left(S_{x}, S_{y}, S_{z}\right) \rightarrow\left(-S_{x}, S_{y},-S_{z}\right),
$$

and a three-fold rotation $C_{3 z}$ which consists of $\pi / 3$ rotation around the $z$ axis:

$$
C_{3 z}:\left(S_{x}, S_{y}, S_{z}\right) \rightarrow\left(-\frac{1}{2} S_{x}-\frac{\sqrt{3}}{2} S_{y}, \frac{\sqrt{3}}{2} S_{x}-\frac{1}{2} S_{y}, S_{z}\right),
$$

where, $S_{x}, S_{y}$, and $S_{z}$ are the cartesian components of the spin polarization. The reflection $M_{1}$ ensures the vanishing $x$ and $z$ components of spin polarizations, while the three-fold screw rotation $C_{3 z}$ ensures the vanishing $x$ and $y$ components of spin polarizations. Overall, the spin polarization should be zero.

The top valence bands of zinc-blend semiconductors are well described by the $4 \times 4$ Luttinger model expressed in the basis of total angular momentum $j=3 / 2^{33,34}$. We further adopt this method to prove the unusual band splitting with vanishing spin polarization in GaAs (see Supplementary Note 3). The band structure of the effective Hamiltonian along $\Lambda$ line are shown in Fig. 3a. The top valence bands are two non-degenerate bands. The $x$ component of spin polarizations is zero for these nondegenerate bands, as shown in Fig. 3b. The $y$ and $z$ components have the same behavior. This $k \cdot p$ analysis confirms our DFT results and group theory analysis. In Dresselhaus/Rashba effect, the spin polarization of the split bands arises as a consequence of the effective $k$-dependent magnetic field experienced by electrons in the presence of SOC in an acentric environment. When the symmetry requirements unveiled by our group-theory analysis are met, however, the effect of such SOC-induced magnetic fields vanishes, thus giving rise to BSVSP effect. Our further analysis shows indeed that the SOC-induced effective magnetic fields acting on two different $p$ orbitals (e.g., $p_{x}$ and $p_{y}$ ) of the same atom (e.g., As) are equal in strength but opposite in direction for the k-point along the $\Gamma$ - $\mathrm{L}$ direction, resulting in a band splitting and a net vanishing spin polarization (since local magnetic moments for different orbitals of the same atom are opposite) (see Fig. $1 \mathrm{~b}$ and Supplementary Note 3).

Our calculations show that not only the net spin polarization, but also the local spin polarizations of $\mathrm{Ga}$ and As atoms for these two single degenerate bands vanish. This can be understood by symmetry arguments. The site symmetry groups of $\mathrm{Ga}$ and As atoms are $T_{\mathrm{d}}$ and the little group of $\Lambda$ line is $C_{3 \mathrm{v}}$, which is a subgroup of $T_{\mathrm{d}}$. The magnetization of $\mathrm{Ga}$ and As atoms must satisfy the symmetry of $C_{3 \mathrm{v}}$ which is a non-pseudo-polar point group.

We propose that one can tune spin polarization with strain. For example, an in-plane strain applied to GaAs would lower the crystallographic point group to $D_{2 \mathrm{~d}}$. The little point group along symmetry line $\Gamma \rightarrow \mathrm{P}$ (also along [111] direction) is $C_{2}$ which is pseudo-polar. As a consequence, a net spin polarization is symmetry allowed, as indeed confirmed by our DFT calculations when applying an in-plane compressive strain to cubic GaAs (Supplementary Fig. 2).

BSVSP in 2D ferroelectric SnTe. Recently, 2D ferroelectric SnTe with high Curie temperature has been observed ${ }^{35,36}$. This material comprises two sheets of alternating $\mathrm{Sn}$ and $\mathrm{Te}$ atoms, where the in-plane ferroelectric polarization-arising from the relative offset of $S n$ and Te sublattices-breaks the inversion symmetry. Its space group is $P m n 2_{1}$ with $C_{2 \mathrm{v}}$ crystallographic point group, where we
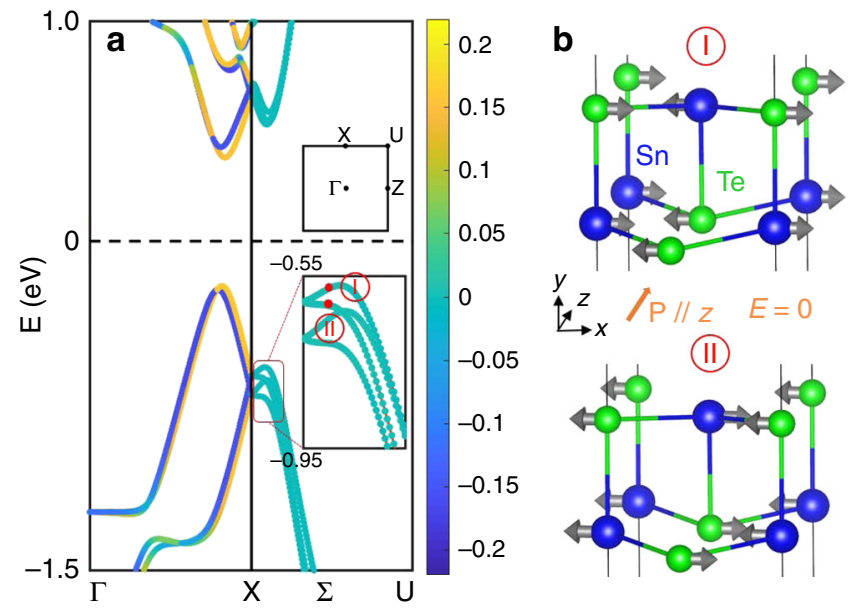

Fig. 4 BSVSP in 2D SnTe thin film. a Band structure and the $y$ components of spin polarizations of 2D SnTe thin film calculated by DFT. Both $x$ and $z$ components vanish and are not shown here. $\mathbf{b}$ Local spin polarizations of Sn and Te atoms for the Bloch states marked with the red points I and II in a. The blue and green spheres are $\mathrm{Sn}$ and Te atoms, respectively

choose the polarization direction as the $z$ axis, while the direction normal to the 2D thin film is taken as the $y$ axis (see Fig. 4b). In Fig. $4 \mathrm{a}$ we show the band structure around the $\mathrm{X}$ point, where four non-degenerate bands are found along the symmetry lines $\Sigma$ $(\mathrm{X} \rightarrow \mathrm{U})$ and $\Delta(\mathrm{X} \rightarrow \Gamma)$, merging in two two-fold degenerate bands at the time-reversal invariant point $\mathrm{X}$, labeled as $\tilde{\mathrm{X}}_{2}, \tilde{\mathrm{X}}_{5}$ and $\tilde{\mathrm{X}}_{3}, \tilde{\mathrm{X}}_{4}$, respectively. Along the $\Sigma$ symmetry line, all components of spin polarization vanish, thus realizing the BSVSP effect. On the other hand, the bands along the $\Delta$ symmetry line are fully spin polarized along the $y$ axis, as expected for a unidirectional Rashba effect, being the bands spin-polarization perpendicular to both $\mathbf{k}$ and ferroelectric polarization. This can be explained by looking at the little groups of the two symmetry lines, following our previous analysis. Along symmetry line $\Sigma$, the little point group is $C_{2 v}$, which is non-pseudo-polar. Therefore, the net spin polarizations vanish. Since the little point group of $\Delta$ symmetry line is the pseudo-polar $C_{\mathrm{s}}$, a non-zero spin polarization is allowed along this line. We further construct wave functions using representation theory to prove the vanishing spin polarizations along the symmetry line $\Sigma$ (see Supplementary Note 6). A minimal $k \cdot p$ model can be written for the valence top bands around $\mathrm{X}$, which reads as $H=-\Delta \tau_{y} \sigma_{y}-\alpha_{B}\left(k_{z} \tau_{z} \sigma_{x}-k_{x} \tau_{z} \sigma_{z}\right)+\beta_{R} k_{x} \tau_{0} \sigma_{y}+\alpha_{R} k_{x} \tau_{y} \sigma_{0}$

where $2 \Delta$ is the SOC induced splitting at X, $\alpha_{\mathrm{B}}\left(\alpha_{\mathrm{R}}\right.$ and $\left.\beta_{\mathrm{R}}\right)$ measure the strength of BSVSP (Rashba-like effect), $\sigma$ and $\tau$ are Pauli matrices representing the spin and pseudospin degrees of freedom, respectively (the latter spanning the two-dimensional single-valued representation $\tilde{\mathrm{X}}_{1}$, see Supplementary Note 6). The $k \cdot p$ model also predicts the BSVSP effect.

Spin polarizations along the $\Sigma$ symmetry line projected on $\mathrm{Sn}$ and Te atoms are shown in Fig. 4b. For each atom, only the $x$ component of the spin polarization does not vanish. This is rationalized by considering the site symmetries of $\mathrm{Sn}$ and $\mathrm{Te}$ atoms. The intersection of site symmetry groups $\left(C_{s}\right.$, comprising a $M_{y z}$ mirror operation about the $x=0$ plane) and little point group of $\Sigma$ line $\left(C_{2 \mathrm{v}}\right)$ is $C_{\mathrm{s}}$ which is pseudo-polar. Therefore, there are spin polarizations on each atom. Only the $x$ components survive as the glide reflection $M_{y z}$ results in the zero $y$ and $z$ components. However, local spin polarizations projected on different $\mathrm{Sn}$ and Te atoms have the same values but opposite sign. They cancel each other so the total spin polarizations vanish. Furthermore, all local spin polarizations are reversed in the conjugate split band. This behavior can be regarded as 

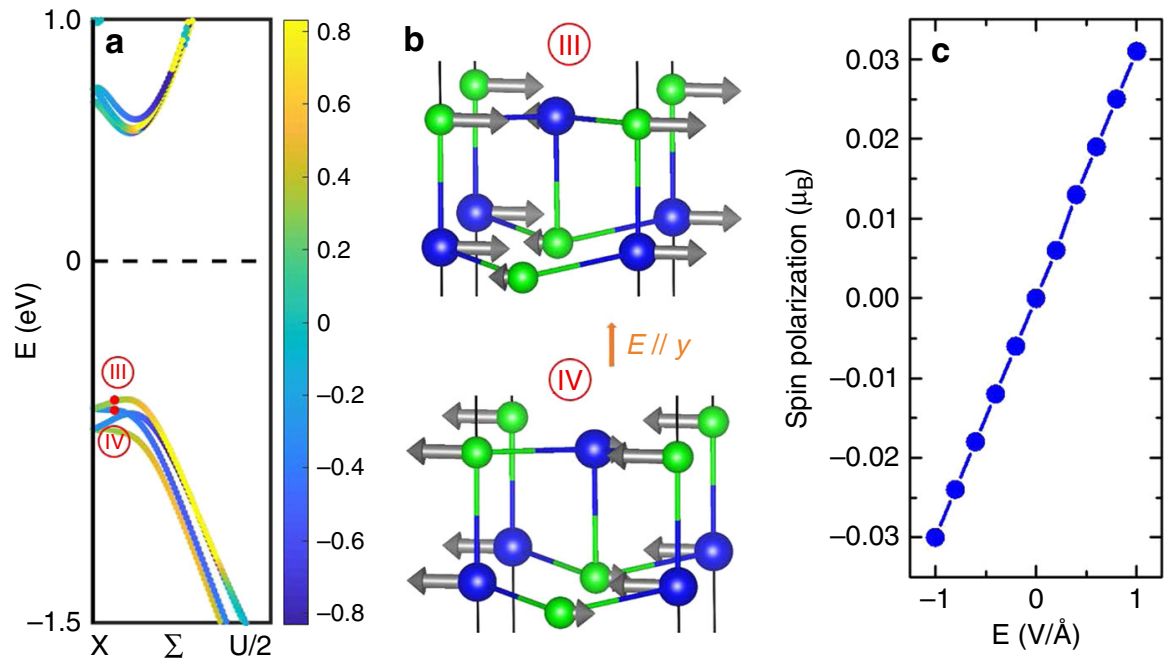

Fig. 5 Linear magnetoelectric coupling in reciprocal space in 2D SnTe thin film. a The $x$ components of spin polarizations when a $0.1 \mathrm{~V} / \AA$ external electric field parallel to $y$ axis (i.e., normal to the film) is added. The vanishing $y$ and $z$ components are not shown. $\mathbf{b}$ Local spin polarizations of Sn and Te atoms for the Bloch states marked with the red points III and IV in a. c Dependence of $S_{x}$ on the external electric field for the Bloch state marked with the red point III

"antiferromagnetism of a Bloch state". The antiferromagnetic order satisfies the little space group symmetry of the highsymmetry line $\Sigma$. In $2 \mathrm{D}$ SnTe, the magnetic fields acting on different atoms of the same kind (e.g., Sn) are opposite in direction for the k-point along the $\mathrm{X}-\mathrm{U}$ direction, resulting in a band splitting and a net vanishing spin polarization (since local magnetic moments for the same kind atom at different positions are opposite) (see Fig. 1b and Supplementary Note 6). The presence of a staggered k-dependent magnetic field along the X-U direction clearly appears in the $k \cdot p$ model restricted to the $\Sigma$ line, which can be recast as $H=-\Delta \tau_{y} \sigma_{y}+B_{x}(k) \sigma_{x}$, where $B_{x}(k)=-\tau_{z} \alpha_{B} k_{z}$.

Tuning of spin polarization with electric field. Previously, it was proposed that the electric field can switch the electric polarization, thus tune the spin texture ${ }^{21,37-39}$. In the 2D SnTe case, the switching of the in-plane electric polarization by $90^{\circ}$ or $180^{\circ}$ by electric field can also result in a change of the spin texture. Here, we rather propose another electric field effect. Since the BSVSP effect is protected by symmetries (e.g., mirror plane), one can use an electric field to break these symmetries and induce a net spin polarization. For example, the SnTe vanishing spin polarizations are protected by the two reflections, $y=0$ and $x=0$ planes. Applying an external electric field along the $y$ axis (normal to the $2 \mathrm{D}$ thin film), which can break the glide reflection $y=0$ plane and reduce the symmetry of $2 \mathrm{D}$ SnTe thin film to the pseudo-polar $C_{\mathrm{s}}$ symmetry, results in the emergence of the $x$ component of spin polarizations. As shown in Fig. 5a, the dependence of the $x$ components on $k$ by applying a $0.1 \mathrm{~V} / \AA$ external electric field indeed confirms our analysis. By projecting spin polarizations on atoms, one can observe that the $x$ components of spin polarizations with different orientations do not cancel each other any more, as shown in Fig. 5b. This behavior can be regarded as "ferrimagnetism of the Bloch state" and ensures the emergence of spin polarizations. The magnitude of spin polarization at a $k$ point is linearly proportional to the external electric field, as shown in Fig. 5c. In particular, an opposite external electric field can lead to a flop of spin polarizations, as shown in Supplementary Fig. 5. This can be understood since these two states are related by the glide reflection-y $=0$ plane. Our $k \cdot p$ model analysis shows that this electric field effect arises from the fact that the electric field causes an energy splitting of the orbitals located at different positions along the out-of-plane direction (see Supplementary Note 7). In some sense, this effect can be considered as one type of linear magnetoelectric coupling ${ }^{40}$, namely linear magnetoelectric coupling in reciprocal space. We recall that in the conventional linear magnetoelectric coupling, the electric field induces a change of magnetization in the whole system. On the contrary, in the linear magnetoelectric coupling in reciprocal space hereby proposed, the electric field induces a change of spin polarization for some particular Bloch eigenstates.

The BSVSP effect might lead to some spintronic applications. By applying strain (e.g., via a piezoelectric material) or electric field to the system displaying the BSVSP effect, the spin polarization can be adjusted, thus the spin transport behavior can be tuned, which might lead to spintronic devices (such as spin-FET ${ }^{41-44}$ ).

\section{Discussion}

In summary, we put forward a phenomenon (dubbed as BSVSP) in nonmagnetic inversion-asymmetric systems: band splitting induced by inversion-symmetry breaking, while the net spin polarization is zero. This phenomenon is rationalized in terms of protection by non-pseudo-polar symmetries. The BSVSP behavior can occur not only in symmorphic but also in nonsymmorphic systems, as shown by our density functional theory calculations demonstrating that BSVSP takes place in bulk GaAs and 2D ferroelectric SnTe. BSVSP is not only interesting from the physics point of view, but also promising for applications. For instance, we propose a linear magnetoelectric coupling in reciprocal space, which can be adopted to tune the spin polarizations of Bloch states by means of an external electric field.

\section{Methods}

DFT calculations. To compute the band structure, our first-principles density functional theory (DFT) calculations are performed on the basis of the projector augmented-wave method ${ }^{45}$ encoded in the Vienna ab initio simulation package $(\text { VASP })^{46,47}$ using the generalized-gradient approximation (GGA) of Perdew et al. ${ }^{48}$. The plane-wave cutoff energy was set to $400 \mathrm{eV}$. A $12 \times 12 \times 12(12 \times 12 \times$ 1) Monkhorst-Pack $k$-mesh is employed to sample the Brillouin zone of bulk GaAs (2D SnTe thin film).

\section{Data availability}

The data that support the findings of this study are available from the corresponding authors upon reasonable request. 
Received: 21 March 2019; Accepted: 25 October 2019;

Published online: 13 November 2019

\section{References}

1. Manchon, A., Koo, H. C., Nitta, J., Frolov, S. M. \& Duine, R. A. New perspectives for Rashba spin-orbit coupling. Nat. Mater. 14, 871-882 (2015).

2. Fabian, J. \& Das Sarma, S. Spin relaxation of conduction electrons. J. Vac. Sci. Technol. B 17, 1708-1715 (1999).

3. Averkiev, N. S. \& Golub, L. E. Giant spin relaxation anisotropy in zinc-blende heterostructures. Phys. Rev. B 60, 15582-15584 (1999).

4. Meier, F. \& Zakharchenya, B. P. Optical Orientation (Elsevier, 2012).

5. Hirsch, J. E. Spin Hall effect. Phys. Rev. Lett. 83, 1834-1837 (1999).

6. Sinova, J. et al. Universal intrinsic spin Hall effect. Phys. Rev. Lett. 92, 126603 (2004).

7. Sinova, J., Valenzuela, S. O., Wunderlich, J., Back, C. H. \& Jungwirth, T. Spin Hall effects. Rev. Mod. Phys. 87, 1213-1259 (2015).

8. Wunderlich, J., Kaestner, B., Sinova, J. \& Jungwirth, T. Experimental observation of the spin-Hall effect in a two-dimensional spin-orbit coupled semiconductor system. Phys. Rev. Lett. 94, 047204 (2005).

9. Schliemann, J. Colloquium: persistent spin textures in semiconductor nanostructures. Rev. Mod. Phys. 89, 011001 (2017).

10. Koralek, J. D. et al. Emergence of the persistent spin helix in semiconductor quantum wells. Nature 458, 610-613 (2009).

11. Sasaki, A. et al. Direct determination of spin-orbit interaction coefficients and realization of the persistent spin helix symmetry. Nat. Nanotech. 9, 703-709 (2014).

12. Zhang, X., Liu, Q., Luo, J.-W., Freeman, A. J. \& Zunger, A. Hidden spin polarization in inversion-symmetric bulk crystals. Nat. Phys. 10, 387-393 (2014).

13. Ganichev, S. D. et al. Spin-galvanic effect. Nature 417, 153-156 (2002).

14. Ganichev, S. D. Spin-galvanic effect and spin orientation by current in nonmagnetic semiconductors. Int. J. Mod. Phys. B 22, 1-26 (2008).

15. Dresselhaus, G. Spin-orbit coupling effects in zinc blende structures. Phys. Rev. 100, 580-586 (1955).

16. Bychkov, Y. A. \& Rashba, E. I. Properties of a 2D electron gas with lifted spectral degeneracy. J. Exp. Theor. Phys. Lett. 39, 78-81 (1984).

17. Bychkov, Y. A. \& Rashba, E. I. Oscillatory effects and the magnetic susceptibility of carriers in inversion layers. J. Phys. 17, 6039-6045 (1984).

18. Rashba, E. I. Properties of semiconductors with an extremum loop. 1. Cyclotron and combinational resonance in a magnetic field perpendicular to the plane of the loop. Sov. Phys. Solid State 2, 1109-1122 (1960).

19. Ishizaka, K. et al. Giant Rashba-type spin splitting in bulk BiTeI. Nat. Mater. 10, 521-526 (2011).

20. Bahramy, M. S., Arita, R. \& Nagaosa, N. Origin of giant bulk Rashba splitting: application to BiTeI. Phys. Rev. B 84, 041202 (2011).

21. Picozzi, S. Ferroelectric Rashba semiconductors as a novel class of multifunctional materials. Front. Phys. 2, 10 (2014).

22. Liebmann, M. et al. Giant Rashba-type spin splitting in ferroelectric GeTe (111). Adv. Mater. 28, 560-565 (2016).

23. Rinaldi, C. et al. Ferroelectric control of the spin texture in GeTe. Nano Lett. 18, 2751-2758 (2018).

24. Stroppa, A. et al. Tunable ferroelectric polarization and its interplay with spinorbit coupling in tin iodide perovskites. Nat. Commun. 5, 5900 (2014).

25. Kuschel, T. \& Reiss, G. SPIN ORBITRONICS Charges ride the spin wave. Nat. Nanotech. 10, 22-24 (2015).

26. Riley, J. M. et al. Direct observation of spin-polarized bulk bands in an inversion-symmetric semiconductor. Nat. Phys. 10, 835-839 (2014).

27. Bawden, L. et al. Spin-valley locking in the normal state of a transition-metal dichalcogenide superconductor. Nat. Commun. 7, 11711 (2016).

28. Yao, W. et al. Direct observation of spin-layer locking by local Rashba effect in monolayer semiconducting $\mathrm{PtSe}_{2}$ film. Nat. Commun. 8, 14216 (2017).

29. Santos-Cottin, D. et al. Rashba coupling amplification by a staggered crystal field. Nat. Commun. 7, 11258 (2016).

30. Slawinska, J., Narayan, A. \& Picozzi, S. Hidden spin polarization in nonmagnetic centrosymmetric $\mathrm{BaNiS}_{2}$ crystal: Signatures from first principles. Phys. Rev. B 94, 241114 (2016).

31. Wu, S.-L. et al. Direct evidence of hidden local spin polarization in a centrosymmetric superconductor $\mathrm{LaO}_{0.55} \mathrm{~F}_{0.45} \mathrm{BiS}_{2}$. Nat. Commun. 8, 1919 (2017).

32. Gotlieb, K. et al. Revealing hidden spin-momentum locking in a hightemperature cuprate superconductor. Science 362, 1271-1275 (2018).

33. Luttinger, J. M. Quantum theory of cyclotron resonance in semiconductors: general theory. Phys. Rev. 102, 1030-1041 (1956).

34. Pidgeon, C. R. \& Groves, S. H. Inversion-asymmetry and warping-induced interband magneto-optical transitions in InSb. Phys. Rev. 186, 824-833 (1969).
35. Chang, K. et al. Discovery of robust in-plane ferroelectricity in atomic-thick SnTe. Science 353, 274-278 (2016).

36. Liu, K., Lu, J., Picozzi, S., Bellaiche, L. \& Xiang, H. Intrinsic origin of enhancement of ferroelectricity in SnTe ultrathin films. Phys. Rev. Lett. 121, 027601 (2018).

37. Di Sante, D., Barone, P., Bertacco, R. \& Picozzi, S. Electric control of the giant rashba effect in bulk GeTe. Adv. Mater. 25, 509-513 (2013).

38. da Silveira, L. G. D., Barone, P. \& Picozzi, S. Rashba-Dresselhaus spin-splitting in the bulk ferroelectric oxide $\mathrm{BiAlO}_{3}$. Phys. Rev. B 93, 245159 (2016).

39. Tao, L. L., Paudel, T. R., Kovalev, A. A. \& Tsymbal, E. Y. Reversible spin texture in ferroelectric $\mathrm{HfO}_{2}$. Phys. Rev. B 95, 245141 (2017).

40. Spaldin, N. A. \& Fiebig, M. The renaissance of magnetoelectric multiferroics. Science 309, 391-392 (2005)

41. Datta, S. \& Das, B. Electronic analog of the electro-optic modulator. Appl. Phys. Lett. 56, 665-667 (1990)

42. Koo, H. C. et al. Control of spin precession in a spin-injected field effect transistor. Science 325, 1515-1518 (2009).

43. Chuang, P. et al. All-electric all-semiconductor spin field-effect transistors Nat. Nanotech. 10, 35-39 (2015).

44. Schliemann, J., Egues, J. C. \& Loss, D. Nonballistic spin-field-effect transistor. Phys. Rev. Lett. 90, 146801 (2003).

45. Blöchl, P. E. Projector augmented-wave method. Phys. Rev. B 50, 17953-17979 (1994).

46. Kresse, G. \& Furthmuller, J. Efficiency of ab-initio total energy calculations for metals and semiconductors using a plane-wave basis set. Comp. Mater. Sci. 6, $15-50$ (1996).

47. Kresse, G. \& Furthmuller, J. Efficient iterative schemes for ab initio total-energy calculations using a plane-wave basis set. Phys. Rev. B 54, 11169-11186 (1996).

48. Perdew, J. P., Burke, K. \& Ernzerhof, M. Generalized gradient approximation made simple. Phys. Rev. Lett. 77, 3865-3868 (1996).

\section{Acknowledgements}

Word at Fudan is supported by NSFC 11825403, the Special Funds for Major State Basic Research (Grant No. 2015CB921700), the Program for Professor of Special Appointmen (Eastern Scholar), the Qing Nian Ba Jian Program, and the Fok Ying Tung Education Foundation. K.L. thanks Junsheng Feng for useful discussions.

\section{Author contributions}

H.X. and S.P. proposed the concept and supervised the project. K.L. performed the firstprinciples calculations with the help from W.L. The group theory and $k \cdot p$ analysis were carried out by K.L. with the help of W.L., J.J. and P.B. K.L. prepared the initial draft of the paper. All authors contributed to the writing and revision of the paper.

\section{Competing interests}

The authors declare no competing interests.

\section{Additional information}

Supplementary information is avaliable for this paper at https://doi.org/10.1038/s41467 019-13197-z.

Correspondence and requests for materials should be addressed to S.P. or H.X.

Peer review information Nature Communications thanks Hena Das, Jun-Wei Luo and the other, anonymous, reviewer(s) for their contribution to the peer review of this work

Reprints and permission information is available at http://www.nature.com/reprints

Publisher's note Springer Nature remains neutral with regard to jurisdictional claims in published maps and institutional affiliations.

Open Access This article is licensed under a Creative Commons Attribution 4.0 International License, which permits use, sharing, adaptation, distribution and reproduction in any medium or format, as long as you give appropriate credit to the original author(s) and the source, provide a link to the Creative Commons license, and indicate if changes were made. The images or other third party material in this article are included in the article's Creative Commons license, unless indicated otherwise in a credit line to the material. If material is not included in the article's Creative Commons license and your intended use is not permitted by statutory regulation or exceeds the permitted use, you will need to obtain permission directly from the copyright holder. To view a copy of this license, visit http://creativecommons.org/ licenses/by/4.0/

(C) The Author(s) 2019 